\title{
Global Characteristics of the Envelope of Family of Trajectories in Resistive Media
}

\author{
Haiduke Sarafian \\ The Pennsylvania State University, University College, York, USA \\ Email: has2@psu.edu
}

How to cite this paper: Sarafian, H. (2020) Global Characteristics of the Envelope of Family of Trajectories in Resistive Media. American Journal of Computational Mathematics, 10, 431-440.

https://doi.org/10.4236/ajcm.2020.103024

Received: August 4, 2020

Accepted: September 6, 2020

Published: September 9, 2020

Copyright (c) 2020 by author(s) and Scientific Research Publishing Inc. This work is licensed under the Creative Commons Attribution International License (CC BY 4.0).

http://creativecommons.org/licenses/by/4.0/ (c) (i) Open Access

\begin{abstract}
In our recent article [1], we discussed the universal geometric characteristics of the envelope of family of trajectories of projectiles projected with the same speeds and different velocities in a vertical plane under the sole influence of gravity; our current investigation is its natural extension. As shown in [1] even for the simplest case where gravity is the only acting external agent literature overlooked reveling the characteristics of the envelope such as its arc-length, the surface area of the enclosed surface and etc. Calculation leading to these has carried out mostly longhand [1]. The current extended version embodies a realistic scenario where the projectiles in addition to gravity encounter linear velocity-dependent media resistance. In order to fulfil objectives similar to [1], we develop two distinct strategies obtaining the analytic equation for the envelope. On one hand, we solve the equations of motion applying traditional longhand approach. On the other hand, we adopt a Computer Algebra System (CAS), e.g. Mathematica [2] [3]. Having these outputs at hand, via mixed-mode calculation-some longhand and some via CAS-we explore its global geometric characteristics such as its arc-length, the surface area of the enclosure. Because of the calculation complexities we could not have achieved our set goals.
\end{abstract}

\section{Keywords}

Velocity-Dependent Resistive Media, Envelope of Trajectories, Computational Mathematics and Physics, Mathematica

\section{Introduction, Motivations and Goals}

We consider a point-like massive object of mass $\mathrm{m}$ projected with initial velocity $\boldsymbol{v}_{0}$ with respect to horizon in a vertical plane. Assuming the projectile in addition to gravity encounters linear velocity-dependent resistive force, we depict the quan- 
tities of interest in Figure 1. The projectile is a point-like, effects such as size, spin, buoyant force and Magnus effect all are suppressed. These are considered in [4]. The focus and objective of our work are to bypass these details and obtain information about the envelope of a family of trajectories under aforementioned circumstance. Impact of these effects on the trajectories and formulation of the associated envelope is forthcoming.

We adopt Newtonian mechanics strategy to analyze the problem. Accordingly, the net force that is composed of the forces acting on the projectile of mass $\mathrm{m}$ is subject to $\boldsymbol{F}_{\text {net }}=m \boldsymbol{a}$. Here $\boldsymbol{F}_{\text {net }}=m \boldsymbol{g}+\boldsymbol{F}_{\text {resistive }}$. It is our intention to consider a scenario where the resistive force is a linear velocity-dependent entity. Therefore, $\boldsymbol{F}_{\text {resistive }}=m n \boldsymbol{v}$; where $n$ is an index with dimension $\mathrm{T}^{-1}$, its value adjusts the strength of the resistive force. In the Discussion and Conclusion Sections, we made comments concerning scenarios where depending on the need and the interest, other velocity-dependent models may be considered.

\section{Trajectory Equation; Traditional Longhand Calculation}

Because the projectile traverses a $2 \mathrm{D}$ curve we project the master dynamic equation, $\boldsymbol{F}_{\text {net }}$ along the coordinate axes. Along the $\mathrm{x}$-axis this gives,

$$
\frac{\mathrm{d}}{\mathrm{d} t} v_{x}=-n v_{x},
$$

Integrating Equation (1) with initial condition, $v_{x}(t=0) \equiv v_{0 x}=v_{0} \cos [\theta]$ yields,

$$
v_{x}(t)=v_{0 x} \mathrm{e}^{-n t},
$$

Replacing $v_{x}=\mathrm{d} / \mathrm{d} t x(t)$ in Equation (2), and solving the corresponding ODE with initial condition, $x(t=0)=0$, we arrive at,

$$
x(t)=\frac{1}{n} v_{0 x}\left(1-\mathrm{e}^{-n t}\right) .
$$

Similarly, projection of the master dynamic equation along the $y$-axis gives,

$$
\frac{\mathrm{d}}{\mathrm{d} t} v_{y}=-g-n v_{y}
$$

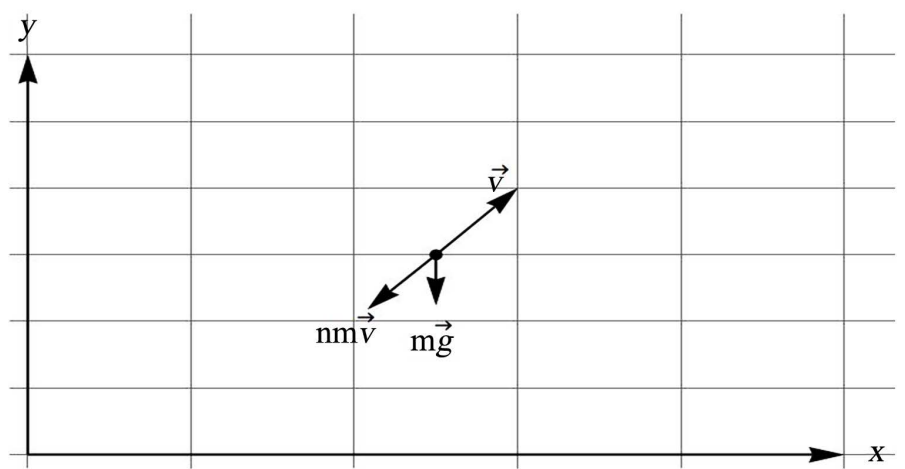

Figure 1. At an arbitrary instant on a vertical plane velocity $\boldsymbol{v}$, gravity pull, $m \boldsymbol{g}$ and media resistance force, $n m v$ are shown. 
Integration Equation (4) and applying the corresponding initial condition, $v_{y}(t=0) \equiv v_{0 y}=v_{0} \sin [\theta]$, gives

$$
v_{y}(t)=\frac{1}{n}\left[-g+\left(g+n v_{0 y} \mathrm{e}^{-n t}\right)\right],
$$

Similar to previous steps we replace $v_{y}=\mathrm{d} / \mathrm{d} t y(t)$ in Equation (5). Solving the corresponding ODE with initial condition, $y(t=0)=0$, yields

$$
y(t)=\frac{1}{n}\left[-g t+\frac{1}{n}\left(g+n v_{0 y}\right)\left(1-\mathrm{e}^{-n t}\right)\right] .
$$

Equations (3) and (6) are explicit time-dependent coordinate of the projectile. Since the objective is to formulate time-independent trajectory equation, utilizing Equation (3) first we write, $E^{-n t}=1-n x / v_{0 x}$ and then solve the latter for $t=-(1 / n) \ln \left(1-n x / v_{0 x}\right)$. Substituting the last two expressions in Equation (6) gives the time-independent equation for the trajectory,

$$
y(x)=\left(\frac{g}{n v_{0 x}}+\tan [\theta]\right) x+\frac{g}{n^{2}} \ln \left(1-n \frac{x}{v_{0 x}}\right),
$$

One of the objectives of our investigation is to focus on the geometric characteristics of the problem on hand. Therefore, without to lose the generality we set the values of the initial speed and the gravity acceleration to unity, i.e. $v_{0}=g=1$. This modifies Equation (7),

$$
y(x)=\left(\frac{1}{n \cos [\theta]}+\tan [\theta]\right) x+\frac{1}{n^{2}} \ln \left(1-\frac{n}{\cos [\theta]} x\right) .
$$

Equation (8) is a parametric, $\theta$-dependent geometric function. It is a non-linear function of $n$. It is an exact equation; no approximation is used deriving it. In reality the value of $n$ is small. Large values of $n$ hamper the projectile's movement; small values of $\mathrm{n}$ are preferred making the scenario practically interesting. In the next subsection we made comments on $n$-dependency of Equation (8). For time being in Figure 2 we have shown two plots of Equation (8) for two values of $n ; n=0.001$, and 0.1 .

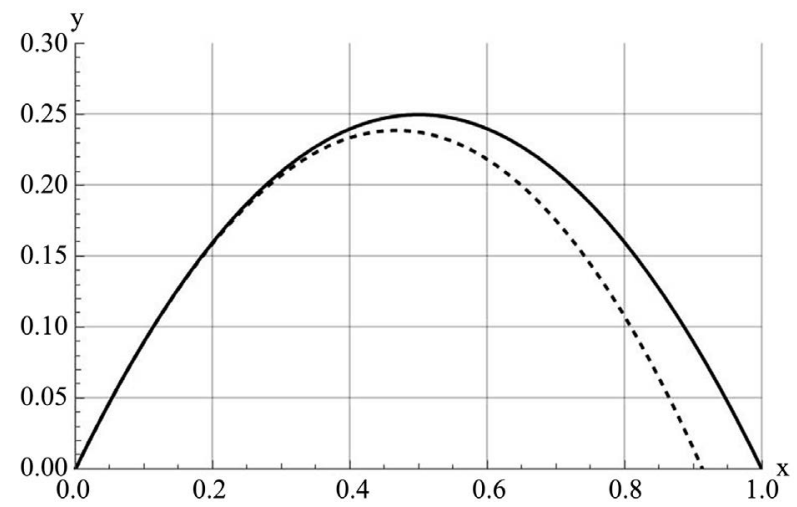

Figure 2. The solid and the dashed curves are the plots of Equation (8). The solid curve with $n=0.001$ is the non-resistive trajectory. The dashed curve is with $n=0.1$. The initial projectile angle for both is set at $\theta=45^{\circ}$. 
According to shown figure as one intuitively would have anticipated the impact of the $n$ is more pronounced beyond the pick point.

Aforementioned procedure is so-called longhand traditional. Utilizing powerful CAS such as Mathematica one may bypass the shown steps short cutting the procedural steps obtaining the same graphic output. Here is what is needed. We write $\{x(t), y(t)\}=\{$ Equation (3), Equation (6) $\}$, and run a one-line command.

Code 1

$$
\begin{aligned}
& \text { Show }\left[\text { Table } \left[\text { ParametricPlot } \left[\left\{\frac{1}{n} v 0 \cos [\theta]\left(1-\mathrm{e}^{-n t}\right),\right.\right.\right.\right. \\
& \left.\frac{1}{n}\left(-g t+\frac{1}{n}(g+v 0 \sin [\theta] n)\left(1-\mathrm{e}^{-n t}\right)\right)\right\} / \text {.values } \\
& / . \theta \rightarrow \frac{\pi}{4},\{t, 0,1.5\}, \text { PlotRange } \rightarrow\{\{0,1 .\},\{0,0.3\}\}, \\
& \text { PlotStyle } \rightarrow \text { Black }],\{n, 0.001,0.1,0.05\}]]
\end{aligned}
$$

The output is identical to Figure 2, we avoid displaying.

\subsection{Trajectory Equation; Computer Algebra System, Mathematica}

Similar to objectives outlines in Section 2.1 utilizing a CAS, specifically Mathematica here we show a short code capable of generating the parametric master Equation (8). The code includes a command, DSolve that symbolically solves two ODEs describing the equations of motions.

Code 2

$$
\begin{aligned}
& \text { initial } x=\left\{x[0]==0, x^{\prime}[0]==v 0 \cos [\theta]\right\} ; \\
& \text { initialy }=\left\{y[0]==0, y^{\prime}[0]==v 0 \sin [\theta]\right\} \text {; } \\
& \text { values }=\{v 0 \rightarrow 1, g \rightarrow 1\} \text {; } \\
& \text { solx }=\text { DSolve }[\{\text { eqx }==0 \text {, initial } x\} / \text {.values, } x[t], t] / / \text { Simplify; } \\
& \text { soly }=\text { DSolve }[\{\text { eqy }==0 \text {, initialy }\} \text { / .values, } y[t], t] / / \text { FullSimplify; } \\
& \text { Flatten }[\{x[t] / \text {.sol } x, y[t] / \text {.soly }\}] \text {; } \\
& x y E q=\text { Eliminate }\left[\begin{array}{l}
\text { Flatten }[\{x==x[t] / \text {.solx }, y==y[t]\}] \\
/ . \text { soly }\}], t] / / \text { FullSimplify; }
\end{array}\right] \\
& (x y E q \llbracket 1,1,2 \rrbracket+x y E q \llbracket 1,2 \rrbracket) / n^{2} / / \text { FullSimplify / / Apart; } \\
& (-x y E q \llbracket 1,1,2 \rrbracket+x y E q \llbracket 1,2 \rrbracket) / n^{2}
\end{aligned}
$$

Interested readers are encouraged to run the code and compare the output vs. Equation (8).

\subsection{Trajectory Equation; Computer Algebra System, Mathematica}

As we shown in previous two sections, irrespective of the chosen methodology, the final exact trajectory equation is given by Equation (8). Also as commented 
the $n$-value controls the media strength. A moderately small $n$-values provide a practical media allowing the projectile traverses on a trajectory. Contrary to a large $n$-value that shortens the trajectory's lifetime mocking its physical features. For reasons cited we consider small $n$-values. Mathematically specking this allows expanding the log term of Equation (8). Expanding the log function to second order, $\mathrm{O}\left(n^{2}\right)$ revives the classic trajectory equation [5].

$$
\begin{aligned}
y 2\left[x_{-}, \theta_{-}\right]= & \left(\left(\frac{g}{(n v 0 \cos [\theta])}+\tan [\theta]\right) x\right. \\
& \left.+\frac{g}{n^{2}} \text { Normal }\left[\text { Series }\left[\log \left[1-\frac{n}{v 0 \cos [\theta]} x\right],\{x, 0,2\}\right]\right)\right)
\end{aligned}
$$

/ / FullSimplify / / Apart

$$
-\frac{g x^{2} \operatorname{Sec}[\theta]^{2}}{2 v 0^{2}}+x \tan [\theta]
$$

And expanding the log piece to the third order, $\mathrm{O}\left(n^{3}\right)$ replaces Equation (8) with,

$$
\begin{aligned}
y 3\left[x_{-}, \theta_{-}\right]= & \left(\left(\frac{g}{(n v 0 \cos [\theta])}+\tan [\theta]\right) x\right. \\
& \left.+\frac{g}{n^{2}} \text { Normal }\left[\text { Series }\left[\log \left[1-\frac{n}{v 0 \cos [\theta]} x\right],\{x, 0,3\}\right]\right)\right)
\end{aligned}
$$

/ / FullSimplify

$$
-\frac{g x^{2}(2 n x+3 v 0 \cos [\theta]) \operatorname{Sec}[\theta]^{3}}{6 v 0^{3}}+x \tan [\theta]
$$

Plots of Equation (8) and (11) for an index value of $n=0.1$ shows indistinguishable trajectories. With this observation instead of applying Equation (8) we utilize its equivalent, i.e. linearized n-dependent format, Equation (12) to developing the envelope equation.

\section{The Envelope}

From this point on we set $n=0.1$ and perform the rest of the calculation utilizing this value only. To generate the analytic equation for the envelope as outlined in [1] we follow the standard procedure. One needs to set the slope of the trajectory equation, i.e. Equation (12) w/parameter, $\theta$, zero, then eliminate the parameter, $\theta$ between the trajectory Equation (12) and the latter. This is the correct recipe however it is tedious and cumbersome. As we shown in detail [1] by introducing a variable such as $\xi=\tan [\theta]$ the procedure simplifies. Applying the $n$-dependent linearized format of the trajectory, i.e. Equation (12), we write,

$$
x \xi-\frac{1}{6 v 0^{3}} g x^{2}\left(1+\xi^{2}\right)^{3 / 2}\left(2 n x+\frac{3 v 0}{\sqrt{1+\xi^{2}}}\right)
$$

We calculate its slope and set it zero, and search its roots, 
Code 3

$$
\begin{aligned}
& \text { slope } \xi=D[\text { eq }[x, \xi],\{\xi, 1\}] ; \\
& \text { sol } \xi 0=\text { Solve }[\text { slope } \xi==0, \xi] ;
\end{aligned}
$$

Solution of this equation is, $\xi=\xi(x)$. After substituting the latter in Equation (12) we arrive at the envelope equation. As one may imagine the final expression is complicated and long. We avoid cluttering the manuscript by not showing its explicit output. Interested readers may run the given code to reveal the explicit envelope equation. Here is the needed line,

Code 4

$$
e q[x, \xi] / . s o l \xi 0 \llbracket 4 \rrbracket / . v a l u e s / . n \rightarrow 0.1 ;
$$

To hone at our objective, we show its corresponding curve, see Figure 3.

By comparing the solid and the dashed black curves one may deduce a few facts. The dashed black curve is what we reported earlier [1]. Nonetheless, we plot this curve utilizing our new n-dependent envelope equation, Equation (12). It is gratifying to observe that this is identical to [1] noting the fact that [1] is based on calculation for a non-resistive media. As anticipated the dashed curve has a higher height than the solid one. This is because the former is for non-resistive media where the solid is for a resistive media. Other interesting information such as the values of maximum heights, maximum abscissa and etc. directly may be read off the graph. More on this on following subsection.

\subsection{Corollary Calculations}

Having the equation of the envelope on hand guided by [1] one may purse similar calculation conducive to some universal information about the envelope. For instance, for a chosen $n$-value e.g. $n=0.001$ and 0.1 applying Mathematica [3] we search for roots of (Equation of envelope) $=0$. As pointed previously the envelope equation is quite lengthy and complicated. By the same token the latter equation is complicated as well. We avoid displaying it, nonetheless we report its numeric value. For two different values of n-index applying Mathematica [3] we arrive at MaxAbscissas: 0.999059 for $n=0.001,0.920814$ for $n=0.1$. These high precision numeric values match the read offs of Figure 3.

\subsection{Arc-Length of the Envelope}

The arc-length of the envelope in principle easily can be calculated. This is subject to $\ell=2 \int_{0}^{x_{a b s c i s s a m a x}} \sqrt{1+\left[\frac{\mathrm{d}}{\mathrm{d} x} y(x)\right]^{2}} \mathrm{~d} x$, here $x_{\text {abscissa }}$ is the maximum value of the abscissa; previous section includes the value. As it was pointed for a comprehensive understanding, we focus on one specific case, $n=0.1$. Bear in mind also that we have formulated the entire analysis symbolically as a function $n$. Meaning, we may produce the on

$$
f i t x=0.99548+0.06049 x+0.46388 x^{2}
$$




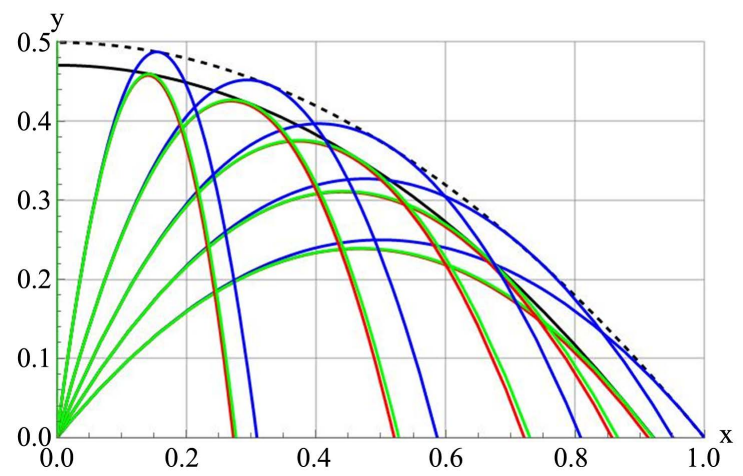

Figure 3. The solid black curve is the envelope of a family of trajectories with resistive index of $n=0.1$. The dashed black curve is the non-resistive envelope for $n=0.001$. The blue parabolas are the family of projectiles projected with the same initial speeds and various velocities, the green curves are the family with same speeds as the blue ones.

going computation for any desired $n$. In practice evaluation of the arc-length, $\ell$, because of complicated expression for $y(x)$ is CPU expensive; my laptop with a quad processor runs forever! To go around this issue utilizing $y(x)$ first we form its symbolic slope, $y^{\prime}(x)$, then we tabulate paired data points, $\left\{x, \sqrt{1+y^{\prime}(x)^{2}}\right\}$ for a handful of $x^{\prime}$. These pairs are shown with dots in Figure 4. Then by applying Mathematica Fit command we fit the data with a second order polynomial. The outcome of the proposed process is depicted in Figure 4 as well. As shown the fitted function is "perfect." Concluding that it is much efficient to replace the integrand with the fitted function.

Integrating Equation (14) over the given limits yields the arc-length of the envelope.

$$
\ell=\int_{0.001}^{0.920814} \text { fit } x \mathrm{~d} x=1.06204
$$

If we could extend the envelope into the second quadrant the arc-length would be twice as long; 2.124 . The arc-length of non-resistive media as reported [1], is $\sqrt{2}+\operatorname{arcsinh}[1]=2.29$, the former falls short by $7.2 \%$. According to Figure 3 this is what one would have anticipated. Meaning, the trajectory of the projectile in the resistive media indexing $n=0.1$ has a shorter abscissa and is lower in height vs. the one for non-resistive media; therefore, its associated arc-length should be shorter as well. As in more than one occasion we pointed out our calculation may be repeated for any desired $n$-value.

\subsection{Surface Area of the Enclosed Surface by the Envelope}

The surface area of the enclosed surface is subject to $s=\int_{0}^{x_{a b s c i s a m a x}} y(x) \mathrm{d} x$. As in the previous case substituting $y(x)$ i.e. the equation the envelope directly in this equation makes the computation CPU expensive. Therefore, we follow the same successful technical procedure of the previous subsection. First utilizing equation of the envelope, we generate a set of data pairs, $\{x, y(x)\}$; these are depicted with dots in Figure 5. Then we fit the data with an appropriate polynomial function. This is shown with the solid curve in the same Figure. Then we 


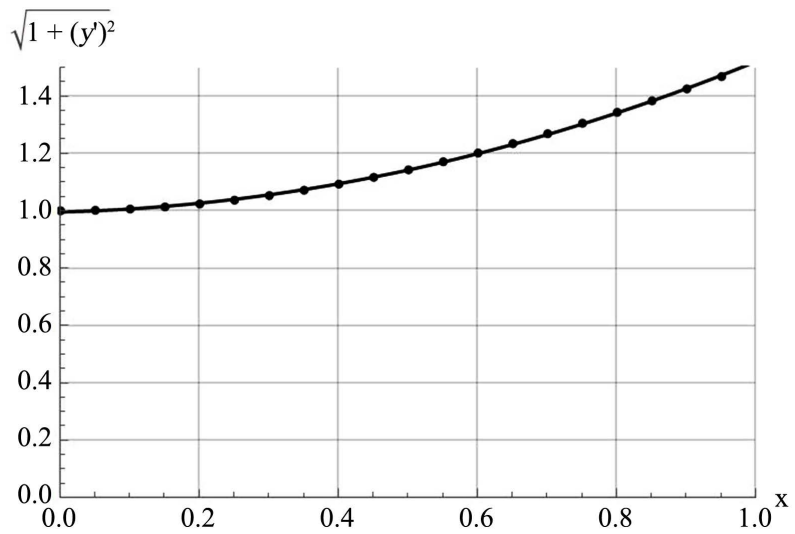

Figure 4. Dots are the paired coordinates, $\left\{x, \sqrt{1+y^{\prime}(x)^{2}}\right\}$. The solid curve is the fitted, fitx, function.

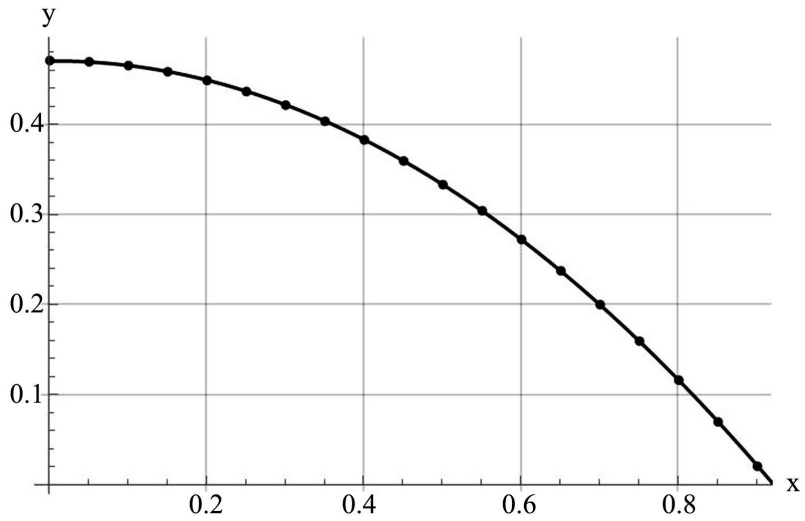

Figure 5. Dots are the paired coordinates of the envelope, $\{x, y(x)\}$. The solid curve is the fitted $\mathrm{x}$-dependent 2 nd order quadratic function.

swap the envelope equation with the fitted polynomial. Equation (15) is the fitted function.

$$
\text { fitxy }=0.4703+0.0072 x-0.5615 x^{2}
$$

Integrating the fitted function, fitxy, yields the surface area enclosed with the envelope.

$$
S=\int_{0.001}^{0.92} f i t x y \mathrm{~d} x=0.28953
$$

By doubling its value, we get 0.579 . This is $1.5 \%$ less than the reported value in [1]. One look at the plotted envelops in Figure 3 justifies the shortcoming. Descriptively speaking we may use the same reasons outlined in the arc-length computation subsection.

\section{Conclusions and Discussions}

In this investigating research article, we pushed the boundaries of our previous work [1] and publications related to the envelope of projectiles in resistive velocity-dependent media. Although the concept of the envelope for a family of tra- 
jectories for projectiles thrown in a vertical plane under the sole action of gravity is familiar, even for such a familiar scenario we report its unknown fresh characteristics [1]. Hence, it is almost inevitable not to extend the analysis for practical situations where in addition to gravity the projectile encounters velocity-dependent resistive media, e.g. movement of microscale bacteria in a viscose media falls in this category.

We introduced two distinct calculational methodologies. For the sake of establishing the basis, first, we produce a set of familiar information, e.g. Equations (3), (6). Then utilizing CAS, specifically Mathematica [3] symbolically we generated the same information and beyond, Equation (8). Utilizing the latter approach, we were able to solve symbolically complicated, some with output as lengthy as a page, equations identifying the velocity-dependent envelope encompassing a family of associated trajectories. As we pointed out in the Abstract without CAS we could not have solved this segment of the project. This is a fresh unreported contribution. Having the equation of the envelope on hand inspired and guided by [1] we explored its geometric global characteristics such as its arc-length and surface area. In doing so because of the CPU expensive computation we introduced a numeric approach fulfilling our goal. The introduced method may be used for similar scenarios.

The author acknowledges despite thorough web-based search ended up empty handed identifying published article(s) addressing the envelope of trajectories in velocity-dependent viscose media.

For interested readers we are suggesting two exercises: computation of the surface area of the revolution of the envelope about its vertical axis, i.e. the surface area of the paraboloid, and its associated volume. Our article embodies sufficient coding information that guided with the computed results [1] these can easily be completed.

For non-microscale objects such as baseball, velocity-dependent resistive forces customarily are formulated as speed-squared forces [4]. As an investigating project based on information provided in our articles [1] [4], one might be interested in pursuing similar analysis. Interested readers on graphics applications of Mathematica will find [6] resourceful.

\section{Acknowledgements}

The author gratefully acknowledges the support of the John T. and Paige S. Smith professorship funds completing this project.

\section{Conflicts of Interest}

The author declares no conflicts of interest regarding the publication of this paper.

\section{References}

[1] Sarafian, H. (2020) Envelope of Family of Angled Projectiles and Its Universal 
Geometric Characteristics. American Journal of Computational Mathematics, 10, 425-430. https://doi.org/10.4236/ajcm.2020.103023

[2] Wolfram, S. (1996) Mathematica Book. 3rd Edition, Cambridge University Press, Cambridge.

[3] Mathematica (2020) https://www.wolfram.com/mathematica/V12.1

[4] Sarafian, H. (2015) Impact of the Drag Force and the Magnus Effect on the Trajectory of a Baseball. World Journal of Mechanics, 5, 49-58. https://doi.org/10.4236/wjm.2015.54006

[5] Halliday, D., Resnick, R. and Walker, J. (2013) Fundamentals of Physics Extended. 10th Ed, John Wiley and Sons, NY.

[6] Sarafian, H. (2019) Mathematica Graphics Examples. 2nd Edition, Scientific Research Publishing. 